\title{
A Case Report of Improvement of Docetaxel-induced Fluid Retention in a Patient with Advanced Gastric Cancer using Herbal Decoction, Modified Bangkihwangki-tang
}

\author{
Seong-Heon Choi ${ }^{1}$, Jee Young Lee ${ }^{1}$, Sung-Un Lee ${ }^{2}$, Soo-Min Lee ${ }^{2}$, Sora Park ${ }^{2}$, \\ Yee-Hong Jung ${ }^{2}$ \\ ${ }^{1}$ Cancer center of Traditional Korean Medicine, Kyung Hee University hospital at Gangdong \\ ${ }^{2}$ Graduate school of Clinical Korean Medicine, Kyung Hee University
}

\begin{abstract}
Objectives: A 54-year-old advanced gastric cancer patient developed severe fluid retention after docetaxel chemotherapy, at cumulative dose of $930 \mathrm{mg} / \mathrm{m}^{2}$, with no improvement using diuretics or prophylactic glucocorticoids. Consequently, he changed chemotherapy regimen from docetaxel to paclitaxel. After he treated with herbal decoction Bangkihwangki-tang (BHT), which is widely used to treat several symptoms including edema in traditional Korean medicine, docetaxel-induced fluid retention (DIFR) was significantly improved along with associated symptoms, such as peripheral edema, pleural and pericardial effusion, pain, scleroderma, and hypoesthesia. As the symptoms were relieved, his performance status and ambulatory ability were improved. During herbal treatment, he continued chemotherapy without any problems and didn't show any adverse events related to herbal medicine. Although there is possibility of natural improvement progress after withdrawal of docetaxel, he showed shorter recovery period and significant improvement despite of severity of initial symptoms.
\end{abstract}

Key Words : Bangkihwangki-tang, docetaxel, fluid retention, gastric cancer, traditional Korean medicine

\section{Introduction}

Docetaxel, a type of taxanes, is widely used to treat breast cancer, non-small cell lung cancer, prostate cancer, gastric cancer, head and neck cancer. Some patients receiving docetaxel develop serious problems with fluid retention which is usually manifest as peripheral edema, pulmonary edema, or pleural effusions ${ }^{1)}$.

Bangkihwangki-tang ("fangiihuangqi-tang" in traditional Chinese medicine; "boiogito" in Kampo medicine), one of representative prescriptions for edema in traditional Korean medicine, is first introduced in ${ }^{『}$ Synopsis of Golden Chamber』, a classic clinical book of traditional Chinese medicine written by Zhang Zhongjing at the end of the Eastern Han Dynasty, and it has been used in Northeast Asia over thousands years to treat several symptoms including edema, obesity, and arthritis. In these days, many investigators conducted research about BHT and reported various effects on edema ${ }^{2-4)}$, obesity ${ }^{5}$, rheumatic arthritis ${ }^{6}$, osteoarthritis ${ }^{6}$, and urinary tract

\footnotetext{
- Received : 24 November 2014

- Revised : 19 December 2014

- Accepted : 19 December 2014

- Correspondence to : Yee-Hong Jung

Graduate school of Clinical Korean Medicine, Kyung Hee University

Tel : +82-2-440-7279, Fax : +82-2-440-7287, E-mail : Adagio554@gmail.com
} 
dysfunction ${ }^{7)}$, etc.

This report presents the case of a patient with advanced gastric cancer that exhibited definite improvement on severe DIFR after treatment with herbal decoction, BHT.

\section{Case Report}

A 54-year-old male patient with advanced gastric cancer with multiple bone metastases was referred with pain, scleroderma, and edema over the both lower legs since two months ago. He was diagnosed with advanced gastric cancer, and then he received total gastrectomy followed by chemotherapy with capecitabine/oxaliplatin (XELOX), leucovorin/fluouracil/ irinotecan (FOLFIRI) regimens and had several times of radiotherapies to metastatic sites. After disease progression, he subsequently received third line chemotherapy with docetaxel with total cumulative dose of $1230 \mathrm{mg} / \mathrm{m}^{2}$. Although docetaxel showed some efficacy to the patient, after four months of receiving docetaxel chemotherapy at cumulative dose of $930 \mathrm{mg} / \mathrm{m}^{2}$, problem of edema at the lower extremities accompanied with pain, scleroderma, and hypoesthesia, gradually deteriorated. Moreover, diuretics could not resolve the edema but caused moderate to severe adverse events such as nausea and vomiting. Furthermore, increased amount of pleural and pericardial effusion was detected by chest x-ray and chest computed tomography (CT) images. As a result, these adverse events made him change the chemotherapy regimen from docetaxel to paclitaxel. Also, his ambulation was possible only with help of wheelchair due to remarkable limitation caused by pain and edema at his lower extremities to move. From his first visit to our clinic, he was treated with herbal decoction BHT, widely used for edema in traditional Korean medicine and has shown hydrostatic modulating effect in several studies.

\section{Treatment and Progress}

At first visit to our hospital, he was unable to walk and showed poor performance status according to Eastern Cooperative Oncology Group Performance Status (ECOG PS) 3, due to grade 3 edema and grade 3 pain according to National Cancer Institute Common Terminology Criteria for Adverse Events (NCI-CTCAE) at his lower legs. The edema caused scleroderma, severe pain, and grade 2 hypoesthesia according to NCI-CTCAE.

The modified BHT (Table 1) was prescribed according to traditional Korean medicine theory to treat edema, and Aconiti Lateralis Preparata Radix was added to cope with feeling of cold at distal extremities. Herbal decoction was administered as liquid form three times a day. In addition, to promote circulation and preservation of $Q i$, non-herbal therapies including acupuncture (LI4, LI11 and SI3, bilaterally), moxibustion (CV4 and CV12), and cupping (Bladder Meridian alongside of the vertebral column, bilaterally) were routinely applied to the patient once a day.

Table 1. The Composition of Modified Bangkihwangki-tang.

\begin{tabular}{cc}
\hline Scientific name & Amount (g/day) \\
\hline Sinomeni Caulis et Rhizoma & 10 \\
Astragali Radix & 10 \\
Atractylodis Rhizoma & 6 \\
Zingiberis Rhizoma Recens & 2 \\
Jujubae Fructus & 2 \\
Glycyrrhizae Radix & 2 \\
Aconiti Lateralis Preparata Radix & 2 \\
\hline
\end{tabular}

After the treatment, the edema had been gradually alleviated without fluctuation of urine volume or influence on electrolyte. Consequently the edema disappeared, and other symptoms such as pain, scleroderma, and hypoesthesia also improved. The improvement of edema was measured by change of circumference of legs and body weight (Figure 1,2). 

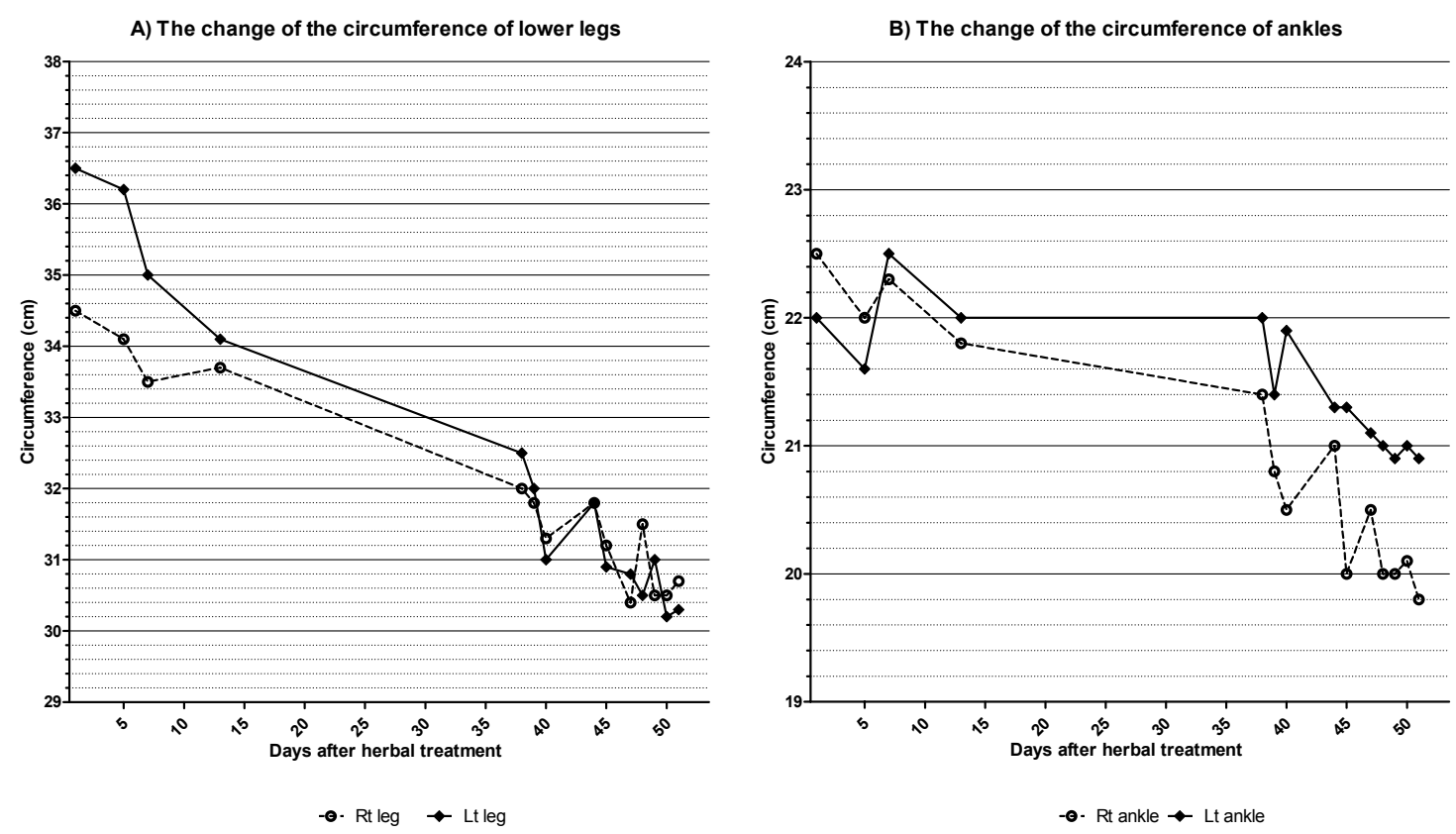

Fig. 1. The change of the circumference of lower extremities.

A) The change of lower legs; at $10 \mathrm{~cm}$ below patella (the widest part at lower leg).

B) The change of ankles; at around medial and lateral epicondyles.

As the symptoms relived, he recovered from bedridden state and could able to walk on foot by himself about 165 meters at a time and the pleural and pericardial effusions has been decreased on radiographic images (Figure 3). Therefore, he was able to receive rehabilitation program to support walking motion and showed improvement in ECOG PS, from 3 to 2 .

While he continued receiving paclitaxel chemotherapy, BHT was additionally administered and, it took him three months to resolve all of the symptoms of the patient without additional use of diuretics or glucocorticoids except chemotherapy premedication. Also, BHT-related adverse effects were not observed. And, his albumin levels were remained stable (approximately $3.5 \mathrm{~g} / \mathrm{dL}$ ).

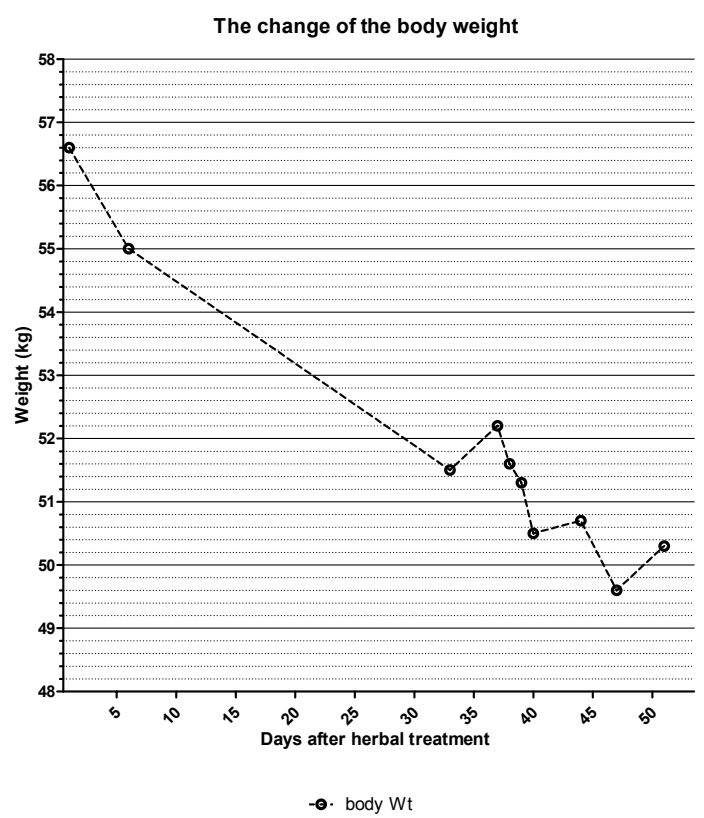

Fig. 2. The change of the body weight 
A) Before herbal treatment

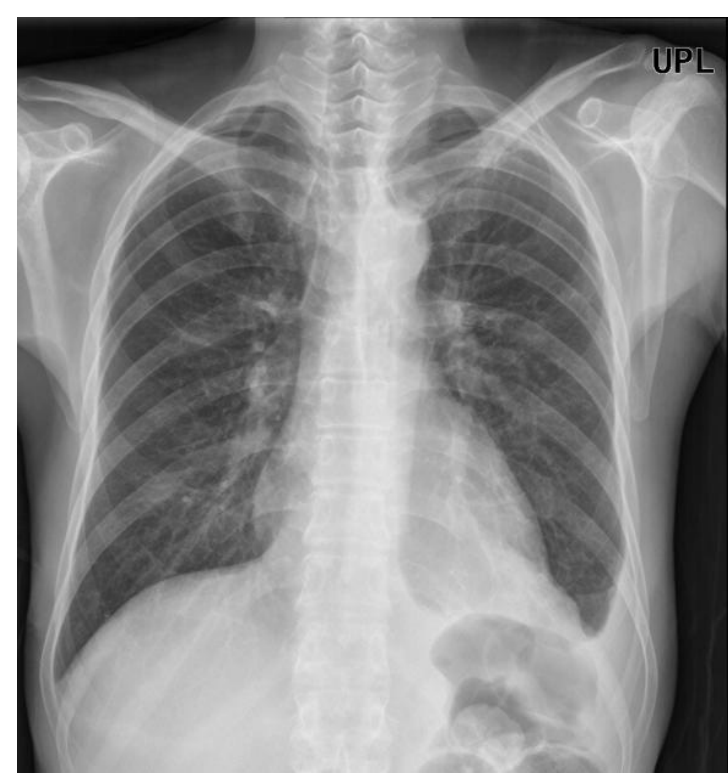

B) After herbal treatment

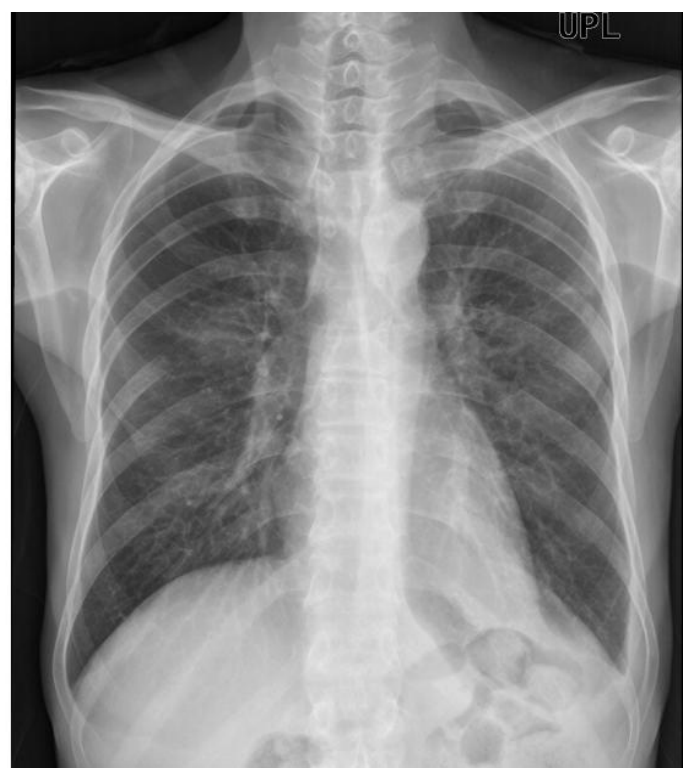

Fig. 3. The change of radiographic images.

Improvement of pleural effusion and cardiomegaly was confirmed on chest $x$-ray images.

A) Before (day 1) and B) After (day 87) administration of herbal decoction, modified Bangkihwangki-tang.

\section{Discussion}

The occurrence of DIFR related to the total dose of docetaxel was administered; it appears at an average cumulative dose of $400 \mathrm{mg} / \mathrm{m}^{2}$ without glucocorticoid premedication. However, in phase II clinical trials, glucocorticoid premedication increased the median cumulative dose to onset of moderate or severe fluid retention from $490 \mathrm{mg} / \mathrm{m}^{2}$ to $790 \mathrm{mg} / \mathrm{m}^{2}$ while reduced the incidence of severe fluid retention from 20 to $6 \%$ and the discontinuation of treatment from 32 to $3 \%{ }^{8,9}$. Most DIFR is reversible after withdrawal of docetaxel (median time to resolution between 16 and 20 weeks), except severe cases in 5 to $6 \%$ of patients ${ }^{10)}$.

The usual initial symptom is peripheral edema and weight gain, and early treatment with diuretics contributes to limiting the severity of DIFR. But continuation of docetaxel chemotherapy with un-controlled fluid retention may progress to pleural and/or pericardial effusions, pulmonary edema, and ascites which may lead to withdrawal of treatment. At first, the appearance of edema was soft pitting pattern and localized at lower part of the body, but it became hard and extended to the whole body disturbing movement of joint after lymphatic involvement ${ }^{11)}$.

Although the underlying mechanism of DIFR is not fully understood, some studies suggest the underlying rationale about pathophysiology of DIFR. In early stage, excessive trans-capillary filtration of proteins (capillary protein leakage) and the primary toxic effects on the capillary endothelium result in congestion of the interstitial space by proteins and waters. Later, mainly after the fifth cycle, insufficiency in lymphatic drainage occurred, inducing clinically obvious fluid retention ${ }^{11,12}$.

Although there was no study about BHT on DIFR, hydrostatic modulating effects of BHT have been reported to control body fluid imbalances in patients suffering from edema. In several in vivo 
studies, "Ohnishi et al." reported moderate diuretic effects of BHT in hypervolemic mice but not in euvolemic mice ${ }^{2}$. "Kim et al." reported that BHT significantly increases urine output via up regulation of renal hemodynamics and down regulation of sodium reabsorption in rabbit $^{3)}$. In clinical study, "Milan et al." reported that administration of BHT in patients with chronic heart failure and renal insufficiency significantly ameliorated renal function, improved estimated glomerular filtration rate $^{4}$. Considering these results, we think BHT as the main component in treat DIFR through hydrostatic modulating effects. Other therapies such as acupuncture, moxibustion, and cupping may be contribute to these hydrostatic modulating effects, but there is not enough evidence about their direct and/or indirect effect on DIFR for now.

In this report, the patient received third line chemotherapy with docetaxel along with prophylactic dexamethasone, a member of glucocorticoid class of steroid drugs. After cumulative dose over 930 $\mathrm{mg} / \mathrm{m}^{2}$, he shows significant clinical manifestation of severe fluid retention, grade 3 according to NCI-CTCAE. Moreover, furosemide and spironolactone, prescribed diuretics for the fluid retention, were not effective, but rather caused moderate to severe nausea and vomiting. As prophylactic steroid and diuretics therapies became unresponsiveness, there is no other way but to stop docetaxel chemotherapy and use other chemotherapy regimen. So, the patient came to our clinic to manage DIFR through traditional Korean medicine.

Since the start of treatment with herbal decoction, BHT, the patient showed subjective improvement such as relief of pain and decrease of scleroderma at his legs. The alleviation of fluid retention was measured with objective methods such as, circumferences around legs, body weight, and radiologic images over a period of time. The recovery time of this patient was 14.5 weeks which is shorter than previously reported median time to recovery from DIFR (median 16-20 weeks ${ }^{10)}$ ). Until now, even though the symptoms almost disappeared, no herbal treatment related adverse event has been reported; he is still continuing the rest of chemotherapy cycles without any severe problems. The immediate improvement of DIFR after applying the herbal treatment and the shortage in the recovery period regarding the severity of the initial symptoms strongly prove the therapeutic potential of BHT.

However, there are several limitations to claim the effect of BHT on DIFR. First, even though the positive reaction of herbal treatments certain, the possibility of natural resolution of DIFR as a result of natural process after withdrawal of docetaxel cannot be ruled out completely. Unfortunately, there is lack of data about natural resolution period after withdrawal of docetaxel in advanced gastric cancer patients. Other study in metastatic breast cancer patient reported median resolution time 16-20 weeks from withdrawal of docetaxe ${ }^{10)}$. Second, the fact that the result was observed only in one patient makes it hard to generalize efficacy of BHT on DIFR.

\section{Summary}

In conclusion, this is the first case report about therapeutic potential of herbal medicine, BHT on DIFR. This result suggests BHT as an alternative option to manage the patients suffering from adverse events of docetaxel chemotherapy. Further clinical experiences and researches on its mechanism will be needed to elucidate the efficacy of BHT on DIFR.

\section{References}

1. Amathieu R, Tual L, Fessenmeyer C, Dhonneur G. Docetaxel-induced acute pulmonary capillary -leak syndrome mimicking cardiogenic oedema. Ann Fr Anesth Reanim. 2007;26(2):180.

2. Ohnishi N, Nagasawa K, Yokoyama T. The 
verification of regulatory effects of Kampo formulations on body fluid using model mice. Journal of Traditional Medicines. 2000;17(3): 131-136.

3. Kim CU, Ryu DG, Lee EJ. The effects of Bangkihwangki-tang on renal functions in rabbit. The Korean Journal of Oriental Medical Prescription. 1990;1(1):183-195.

4. Gautam M, Atsushi I, Tatsuya S, Saeko Y, Hirohiko M, Takeshi T, et al. The Traditional Japanese Medicine (Kampo) Boiogito has a Dual Benefit in Cardiorenal Syndrome: A Pilot Observational Study. Shinshu Med J. 2014;62: 89-97.

5. Park JH, Bose $\mathrm{S}$, Lim CY, Kim HJ. Impact of GNB3, ADRB3, UCP2, and PPAR $\gamma$-Pro12Ala polymorphisms on Boiogito response in obese subjects : A randomized, double-blind, placebo -controlled trial. Journal of Society of Korean Medicine for Obesity Research. 2012;12(2): 28-43.

6. Majima $\mathrm{T}$, Inoue M, Kasahara $\mathrm{Y}$, Onodera $\mathrm{T}$, Takahashi D, Minami A. Effect of the Japanese herbal medicine, Boiogito, on the osteoarthritis of the knee with joint effusion. Sports Med Arthrosc Rehabil Ther Technol. 2012;4(3).
7. Chen G, Liu H, Cheng F. Fangihuangqi tang improved lower urinary tract dysfunction in benign prostatic hyperplasia rats model. J Tradit Chin Med. 2013;33(3):349-54.

8. Cortes JE, Pazdur R. Docetaxel. J Clin Oncol. 1995;13(10):2643.

9. Burris HA. Optimal use of docetaxel (Taxotere): maximizing its potential. Anticancer Drugs. 1996;7 Suppl 2:25-28.

10. Chan S, Friedrichs K, Noel D, Pintér T, Van BS, Vorobiof D, et al. Prospective randomized trial of docetaxel versus doxorubicin in patients with metastatic breast cancer. J Clin Oncol. 1999; 17(8):2341-54.

11. Béhar A, Pujade-Lauraine E, Maurel A, Brun MD, Chauvin FF, Feuilhade de CF, et al. The pathophysiological mechanism of fluid retention in advanced cancer patients treated with docetaxel, but not receiving corticosteroid comedication. Br J Clin Pharmacol. 1997;43(6): 653-8.

12. Semb KA, Aamdal S, Oian P. Capillary protein leak syndrome appears to explain fluid retention in cancer patients who receive docetaxel treatment. J Clin Oncol. 1998;16(10):3426-32. 